\title{
Feasibility Study of Installation of Solar Panels on a High Power HF Antenna Land
}

\author{
Abdul Ali, ${ }^{1}$ Burak Ozbey, ${ }^{1}$ Satilmis Topcu$^{2}$ Ayhan Altintas ${ }^{1}$ \\ ${ }^{1}$ Department of Electrical and Electronics Engineering, Bilkent University, Ankara 06800, Turkey \\ ${ }^{2}$ Bilkent University-Communications and Spectrum Management Research Center (ISYAM), \\ Ankara 06800, Turkey
}

Received 15 December 2015; accepted 9 February 2016

\begin{abstract}
In HF broadcasting, the transmitters are typically very powerful. In the case of Turkish Radio and Television (TRT), total transmission average power may reach up to 5 MW. Therefore, it is natural to utilize solar panels as a means of alternative/backup energy for the continuous transmission of RF signal satisfying the requirement of broadcasting. In the TRT HF broadcasting array field, two areas are designated as the candidate areas for solar panel installation. In these areas, electric fields are simulated, measured, and compared. Simulations are done using the Numerical Electromagnetic Code (4nec2). The suitability of the regions for the solar panel installation are evaluated based on the ICNIRP human exposure limits. In addition, 4nec2 simulations are used to see the effect of metal frames of solar panels on the radiation patterns of the short-wave antenna arrays in the two solar panel candidate areas of TRT HF broadcasting station. Simulation results show that metal frames do not seem to have a significant effect on the radiation patterns of the short-wave antenna arrays. (c) 2016 Wiley Periodicals, Inc. Int J RF and Microwave CAE 26:379_ 384,2016
\end{abstract}

Keywords: short-wave antenna arrays; HF broadcasting; ICNIRP human exposure limits

\section{INTRODUCTION}

International Organizations such as World Health Organization (WHO) and International Commission on NonIonizing Radiation Protection (ICNIRP) determine the human exposure limits from RF radiation that may cause different diseases [1-4]. In Refs. 1-3, ICNIRP declares the upper human exposure limits in terms of time varying electric and magnetic fields for different frequency ranges. The ICNIRP guidelines can play a vital role in determining the areas in the neighborhood of high power HF broadcasting tower where the instantaneous electric field is above the human exposure limits.

When there is a metal object in the vicinity of an antenna, it may cause the properties of the antenna (e.g., return loss, radiation patterns, etc.) to change. In Ref. 5, mutual interference from UHF and VHF antennas on an

Correspondence to: A. Ali; e-mail: abdul@ee.bilkent.edu.tr or A. Altintas; e-mail:altintas@ee.bilkent.edu.tr

DOI: $10.1002 /$ mmce.20975

Published online 23 February 2016 in Wiley Online Library (wileyonlinelibrary.com). existing tower (metal structure) is studied, but it is not studied whether the tower has any effect on the characteristics of the antennas. In Ref. 6, parasitic effects of the tower on characteristics of the antenna such as input impedance, return loss, gain and front-to-back ratio are investigated. Furthermore, in Ref. 7, the effect of a metallic communication tower on the radiation patterns of the antenna is studied, and it is concluded that it depends on frequency, azimuth angle, and elevation angle.

In this article, the effects of short-wave transmitters in the two areas designated for the installation of solar panels are demonstrated through the measurement and the simulation of $E$-fields. The simulations are carried out in 4 nec2 [8]. We define several cases for the measurement of the $E$-field. In each case, some antennas are radiating while others are OFF. The $E$-fields are then compared with the ICNIRP guidelines for the suitability of solar panel installation.

The effect of the metallic frames of solar panels on the radiation patterns of the antenna is also investigated in simulation by comparing the radiation patterns of the antenna in the presence and absence of the metallic

(C) 2016 Wiley Periodicals, Inc. 


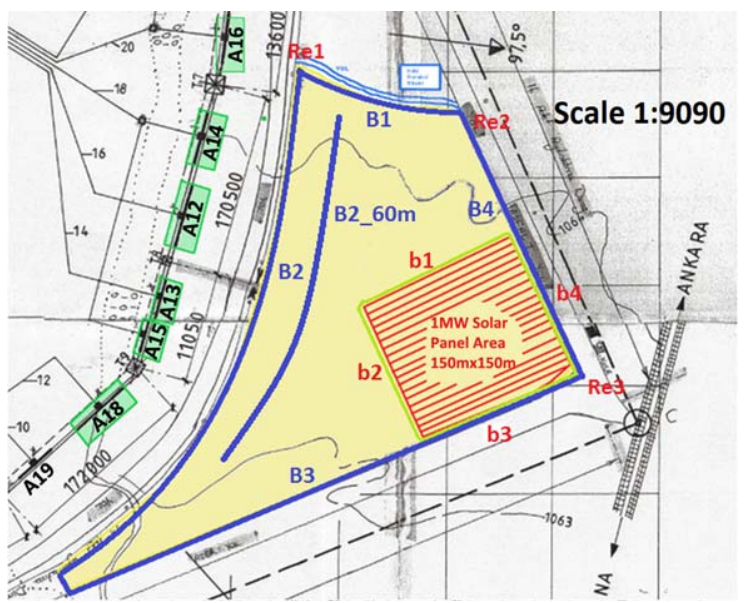

Figure 1 Map showing two candidate areas for solar panel installation.

frames in the two designated areas for solar panel installation.

\section{MEASUREMENTS AND SIMULATIONS}

The short-wave radio transmission station of Turkish Radio and Television (TRT) consists of transmitters, antennas, towers, and transmission lines which are spread over a wide area. It is the location from where radio signals are broadcast to several countries around the world using different frequencies varying in the range 1-30 MHz. The transmitters available in the station are set to operate at different frequencies. These transmitters comprise antenna arrays of different geometries, that are turned $\mathrm{ON}$ and $\mathrm{OFF}$ in a combinational fashion in order to broadcast to a specific country. For a particular antenna, the maximum generator power level is around $500 \mathrm{~kW}$ [9]. In addition to using the electrical grid, cheaper alternative energy resources are sought in order to obtain such a large power. One of the most promising solutions is the use of solar panels. The two regions of interest (yellow and red-shaded) for the installation of solar panels are shown in Figure 1. The transmitting dipole antenna arrays which are predicted to have the most dominant effects on the designated areas are $A_{12}(6-11 \mathrm{MHz})$, $A_{13}(9-17 \mathrm{MHz}), A_{15}(15-26 \mathrm{MHz}), A_{16}(7-13 \mathrm{MHz})$, and $\mathrm{A}_{18}(6-11 \mathrm{MHz})$ (see Fig. 1). In addition, a mechanically controlled rotating antenna $A_{45}(6-11 \mathrm{MHz})$ and a quadrant antenna $A_{53}(7-9 \mathrm{MHz})$ are located further behind dipole arrays (not shown in Fig. 1). $A_{53}$ has an omnidirectional radiation characteristic, while rotating antenna $\left(A_{45}\right)$ is predicted to produce a strong $E$-field when it is pointing toward yellow or red-shaded regions. There is a metal wire curtain between the two antennas and the two regions of interest. The basic element used in antenna arrays is the folded dipole antenna. The antenna $A_{12}$ consists of $2 \times 4$ folded dipole elements. These antenna arrays are studied via simulations and measurements in terms of the level of $E$-field they produce on the yellow and red-shaded regions.
The simulations are done in a freely available software 4nec2 (Numerical Electromagnetic Code) which is based on the Method of Moments (MoM) [8]. The geometry of $A_{12}$ is shown in Figure 2. The height of the antenna from the ground plane is $9 \mathrm{~m}$. The rest of the antennas are similar in shape to $A_{12}$ but with different dimensions. In order to carry out measurement in these two regions, we define several cases in which some of the aforementioned antennas are broadcasting while others are OFF. These cases are listed below:

I. Only $A_{12}$ is $\mathrm{ON}$.

II. Only $A_{45}$ is ON.

III. Only $A_{53}$ is $\mathrm{ON}$.

IV. Only $A_{12}, A_{13}, A_{15}, A_{16}$, and $A_{18}$ are ON.

V. Only $A_{12}, A_{13}, A_{15}, A_{16}$, and $A_{53}$ are $\mathrm{ON}$

VI. Only $A_{12}, A_{13}, A_{15}, A_{16}$, and $A_{45}$ are ON.

VII. Only $A_{12}, A_{13}, A_{15}, A_{45}$, and $A_{53}$ are ON.

Almost all of these cases are investigated in redshaded region with the measurement of the $E$-field, while in the yellow region only the first three cases are considered. The reason for not considering all cases in the yellow region will be clear later in the subsequent section.

\section{A. Yellow Region}

The boundaries of the yellow region are marked with $\mathrm{B} 1$, B2, B3, B4. The measurements are started from reference point $\operatorname{Re} 1$ (for boundaries B1 and B2) as shown in Figure 1. The E-field measurements are performed with a NARDA EMR 300 radiation meter (Probe EF0391 is used which covers the frequency range $100 \mathrm{kHz}$ to $3 \mathrm{GHz}$ ) by using the device on the average value setting. In the first case, when only $A_{12}$ is active, the $E$-field is measured and simulated at some points along the boundary line B2 in the yellow region and then, compared with the ICNIRP human exposure limits. According to ICNIRP guidelines, the human safety limits are $87 / f^{1 / 2} \mathrm{~V} / \mathrm{m}$ and $28 \mathrm{~V} / \mathrm{m}$ RMS (where $f$ is frequency in $\mathrm{MHz}$ ) in the range $1-10 \mathrm{MHz}$ and 10-400 MHz, respectively [1-3]. Table I shows the

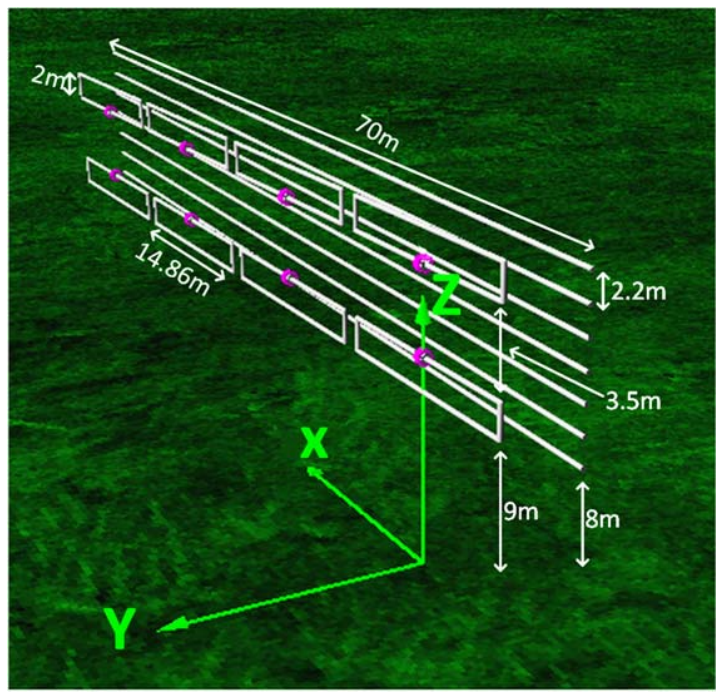

Figure 2 Geometry of $A_{12}$ in 4 nec2. 
TABLE I E-Field Comparison of $\boldsymbol{A}_{12}$ with ICNIRP Limits

\begin{tabular}{lcc}
\hline & \multicolumn{2}{c}{ Percentage (\%) Difference } \\
\cline { 2 - 3 } Point & Measured & Simulated \\
\hline 1 & 27.3 & 87.62 \\
2 & 23.2 & 75 \\
3 & 21.4 & 63.5 \\
4 & 10.7 & 30 \\
\hline
\end{tabular}

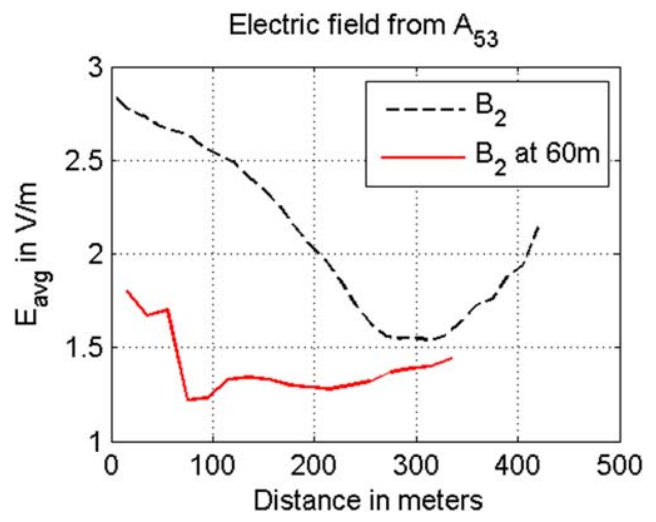

Figure 3 Measured $E$-field of $A_{53}$ in the yellow region.

difference of $E$-field data in percentage when compared with the human exposure limits through Eq. (1).

$$
\% \text { difference }=\frac{E_{\text {experimental }}-28}{28} \times 100
$$

At every point (points 1, 2, 3, and 4 are selected along the boundary line B2) shown in the Table I, the measured and simulated $E$-fields are above the ICNIRP human exposure limits. In the case of $A_{53}$ and $A_{45}$, the measured $E$-fields are shown in Figures 3 and 4, respectively. These plots illustrate that the field strengths from $A_{53}$ and $A_{45}$ in the yellow region are very weak. The fact that there is weak $E$-field is due to the metal curtain between the yellow region and these antennas.

The measured and simulated $E$-fields from $A_{12}$ are observed to be very strong, violating the threshold human exposure limits of ICNIRP. Based on these results, the yel-

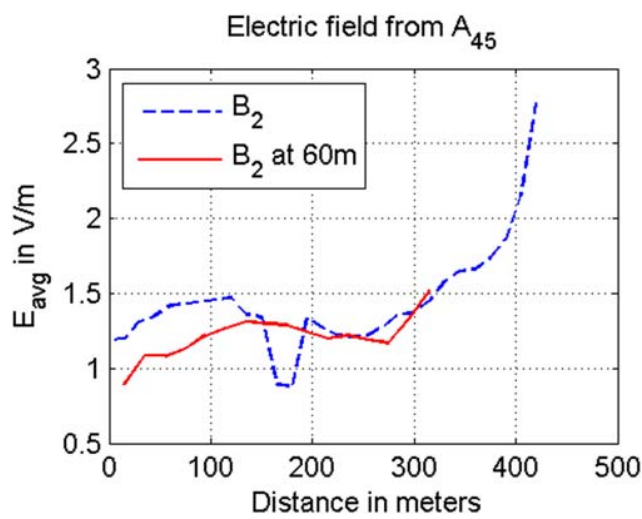

Figure 4 Measured $E$-field of $A_{45}$ in the yellow region.

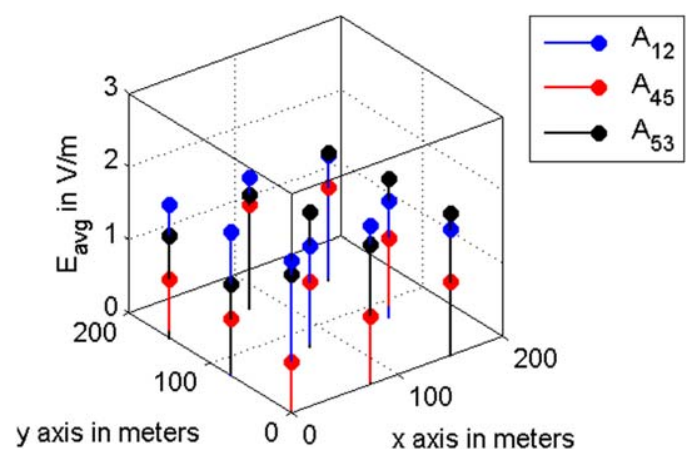

Figure 5 Measured $E$-field of $A_{12}, A_{45}$, and $A_{53}$ in the redshaded region.

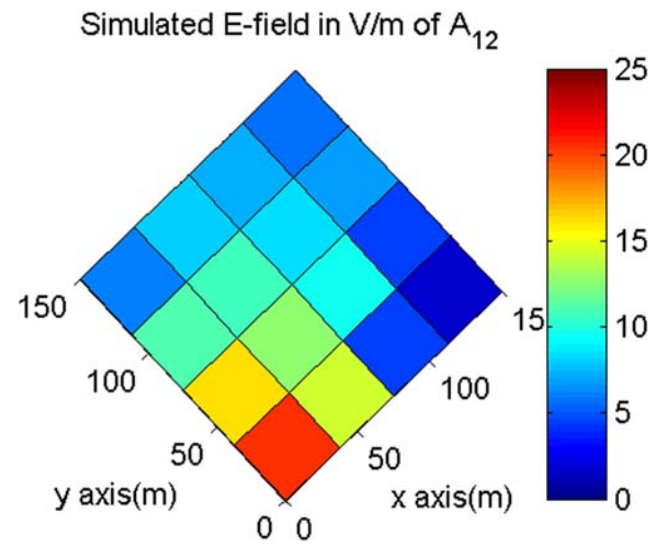

Figure 6 Simulated $E$-field of $A_{12}$ in red-shaded region.

low region is not recommended for the installation of solar panels. Since, the $E$-field from $A_{12}$ is very strong and is by itself above the limits set by ICNIRP, the additional power coming from $A_{13}, A_{15}, A_{16}$, and $A_{18}$ are not studied since, they have a similar geometry and are expected to produce a strong $E$-field above the ICNIRP limits.

\section{B. Red-Shaded Region}

The boundaries of the red-shaded region are marked with b1, b2, b3, b4 and its area is $150 \mathrm{~m} \times 150 \mathrm{~m}$ (see Fig. 1). This region is $175 \mathrm{~m}$ away, which corresponds to

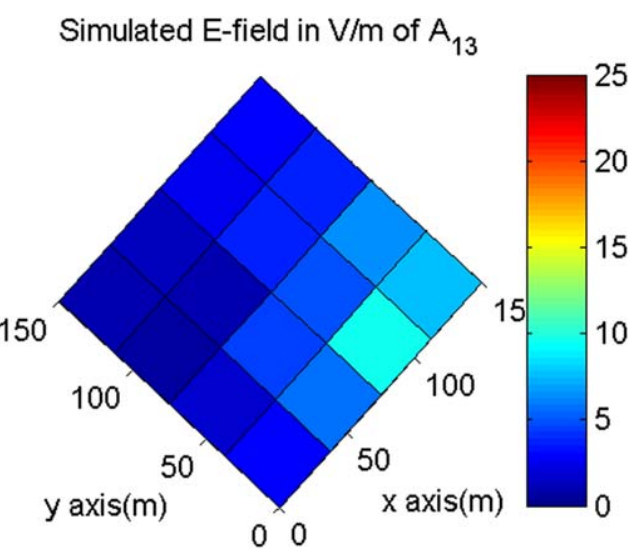

Figure 7 Simulated $E$-field of $A_{13}$ in red-shaded region. 


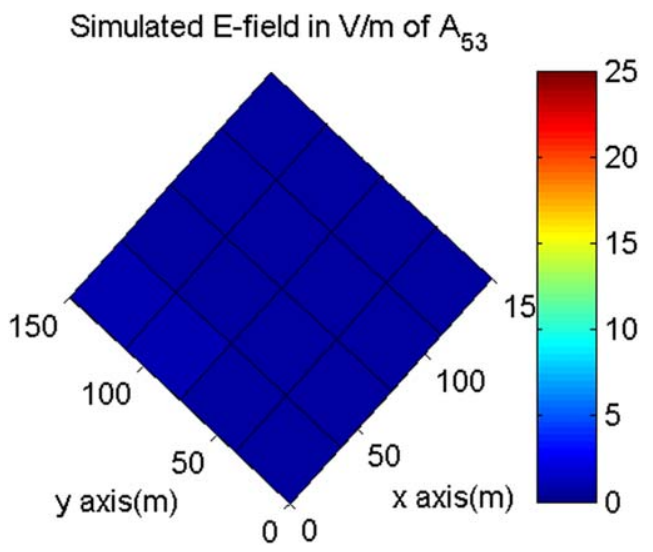

Figure 8 Simulated $E$-field of $A_{53}$ in red-shaded region.

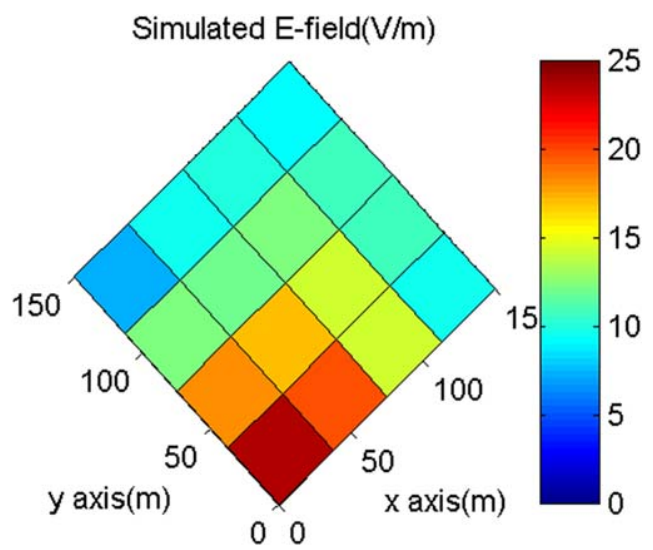

Figure 9 Simulated $E$-field of $A_{12}, A_{13}$, and $A_{53}$ in the redshaded region.

$8.75 \lambda$ when the midpoint of the frequency range is considered $(15 \mathrm{MHz})$. First, the $E$-field from a single transmitter is measured in this region in order to gauge its strength. A step size of $75 \mathrm{~m}$ is used for the first three cases (I, II, III). Figure 5 shows the $E$-field for the first three cases. Figures 6-8 show the simulated $E$-field from $A_{12}, A_{13}$, and $A_{53}$, respectively. Figure 9 shows the total simulated

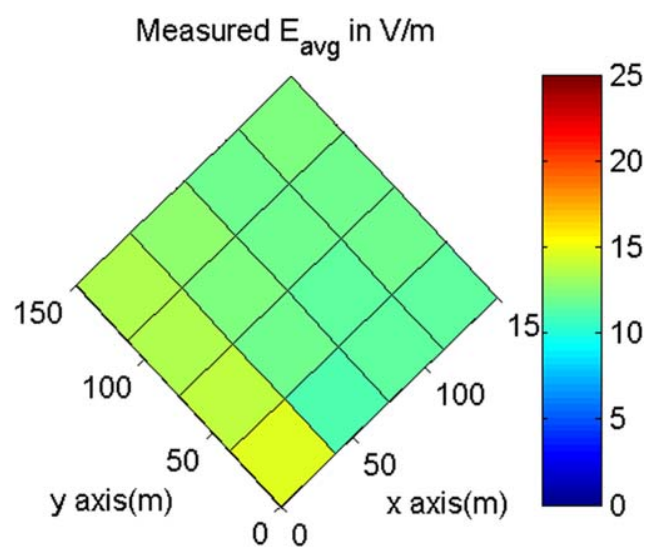

Figure 10 Measured $E$-field of $A_{12}, A_{13}, A_{15}, A_{16}$, and $A_{18}$ in the red-shaded region.

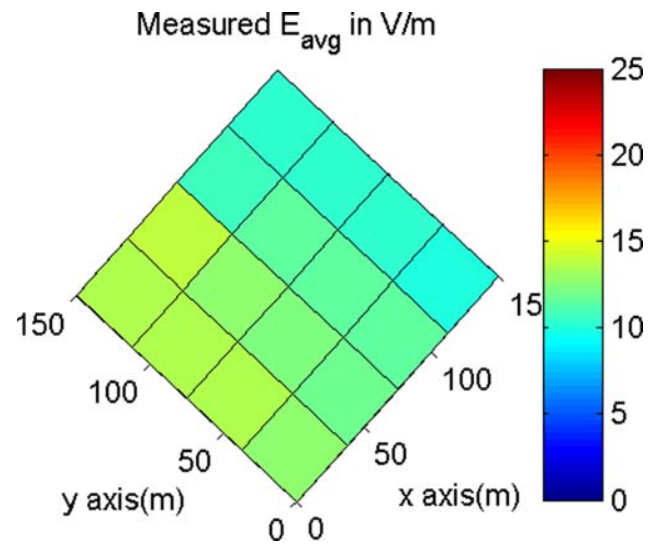

Figure 11 Measured $E$-field of $A_{12}, A_{13}, A_{15}, A_{16}$, and $A_{53}$ in the red-shaded region.

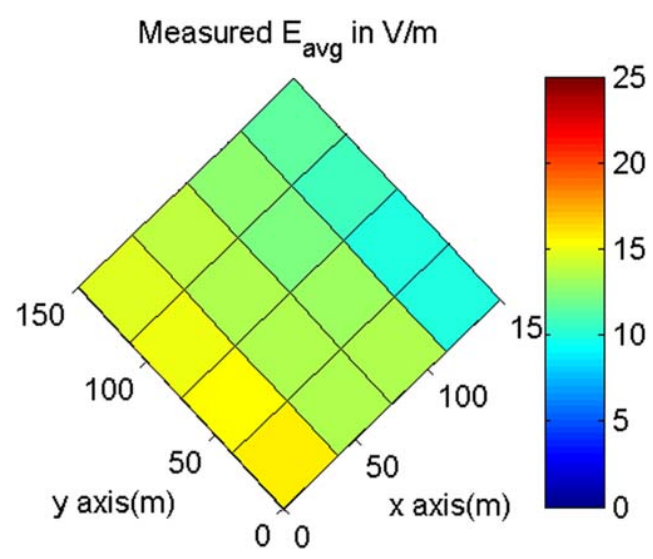

Figure 12 Measured $E$-field of $A_{12}, A_{13}, A_{15}, A_{16}$, and $A_{45}$ in the red-shaded region.

E-field from A12, A13, and A53. The simulation and measurement results show that the $E$-field from a single transmitter is not strong enough to violate ICNIRP human exposure limits. Therefore, it is necessary to inspect the case where there is more than one transmitter to measure the total $E$-field. In the case when more than one

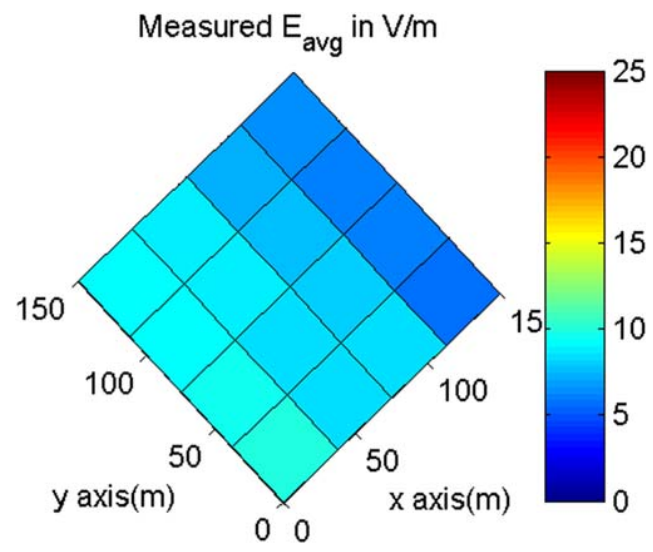

Figure 13 Measured $E$-field of $A_{12}, A_{13}, A_{15}, A_{45}$, and $A_{53}$ in the red-shaded region. 


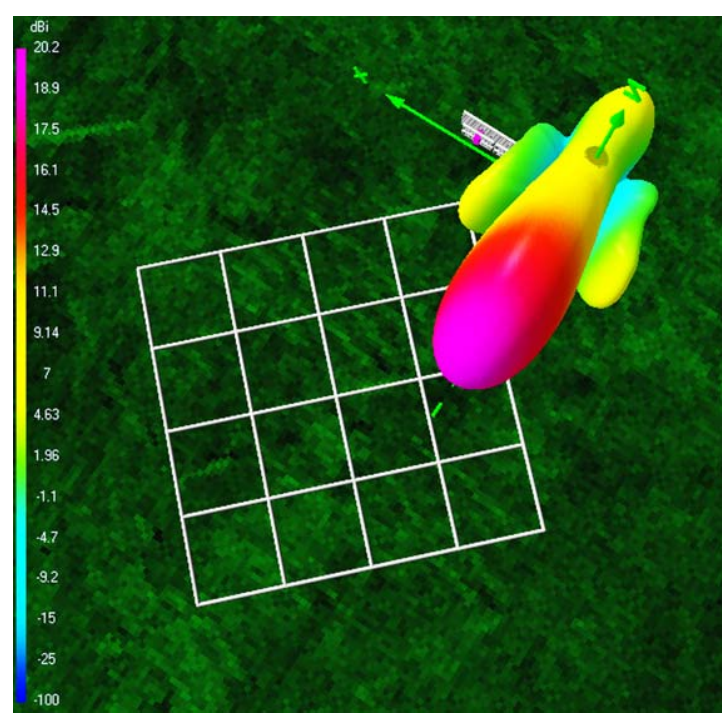

Figure 14 Structure and 3D radiation pattern of $A_{12}$.

transmitter is radiating, $E$-field measurements have been performed with a $30 \mathrm{~m}$ step size in this region. The measured $E$-field from the antennas in Case IV, V, VI, and VII are shown in Figures 10-13, respectively. The measured $E$ field is stronger at the corner of the red-shaded region which is close to the radiating antennas. It can be seen from the figures that the average $E$-field is below the ICNIRP human exposure limits. However, the instantaneous $E$-field may be larger than the threshold values. Moreover, simulated $E$-fields (see Fig. 9) from only three antennas are also stronger at the same corner but slightly below the ICNIRP limits. If the $E$-fields produced by the other antennas are taken into account in the simulation, then the total $E$-field is expected to exceed the ICNIRP limits. The measured and simulated $E$-fields are consistent in terms of field distribution over the red-shaded region.

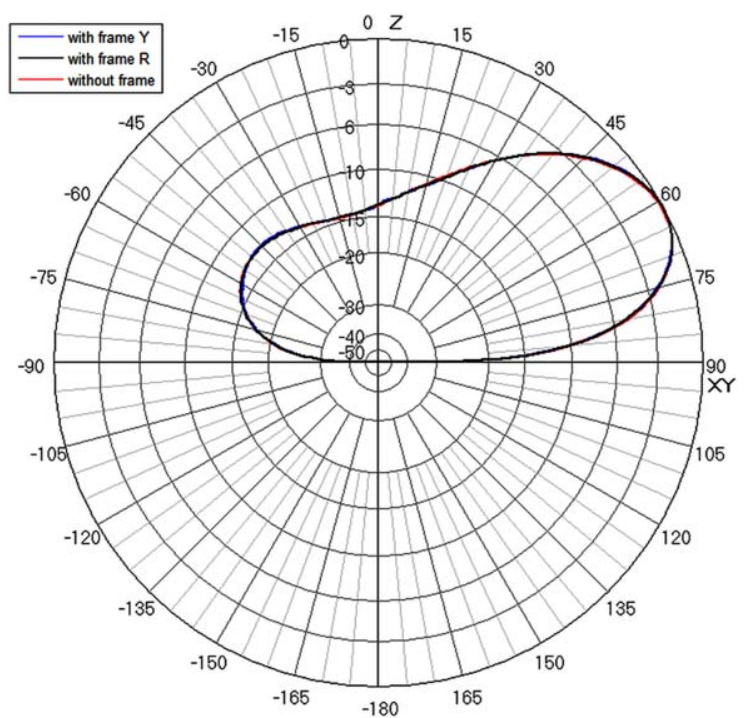

Figure 15 Effect of the solar panel metal frames on the vertical pattern of $A_{12}$.

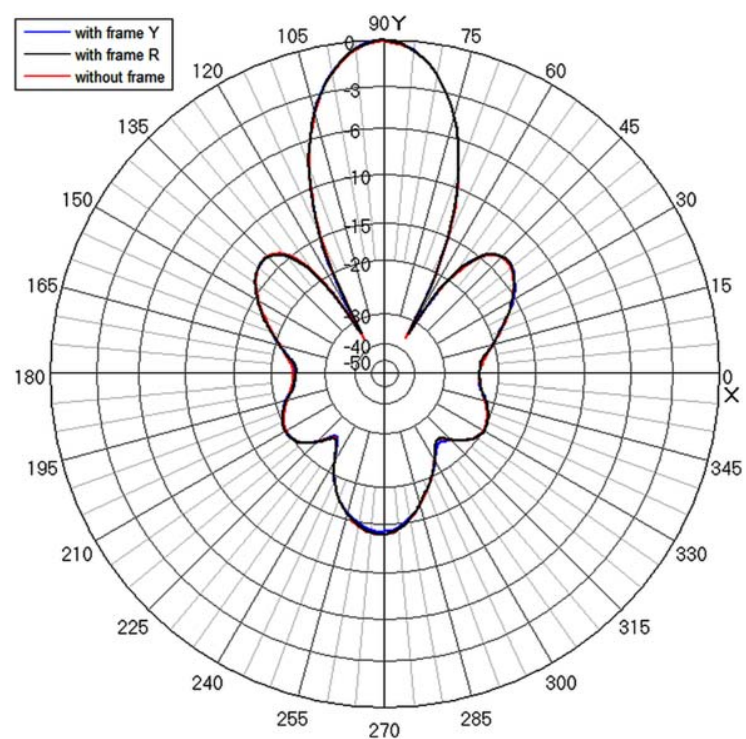

Figure 16 Effect of the solar panel metal frames on the horizontal pattern of $A_{12}$.

However, the measured values are weaker in general. The simulations showing field strengths up to $20 \mathrm{~V} / \mathrm{m}$ are very common. This value is about $3 \mathrm{~dB}$ below ICNIRP limits. If one considers the normal distribution of the field strength with a standard deviation of $6 \mathrm{~dB}$ (ITU assumes $5.5 \mathrm{~dB}$ for broadcasting), then there is a chance of $30 \%$ that the field strength will be above the ICNIRP limits. Thus, the redshaded region is also not recommended in terms of human safety due to the statistical nature of measurements.

\section{Effect of the Solar Panel Metal Frames on Radiation Patterns}

To see the effect of the metal frames on the radiation patterns of the short-wave antenna array, a structure made of wires was created in 4 nec 2 to mimic the frame and it is placed in the yellow and red-shaded regions separately at a height of $2.5 \mathrm{~m}$ from the ground plane. The 3D radiation pattern along with antenna structure and metal frame are shown in Figure 14. The radiation patterns (horizontal and vertical) of the antenna are compared in the case when there is a metal frame in the two regions of interest to the case when there is no metal frame. Figures 15 and 16 show vertical and horizontal radiation patters of the $A_{12}$, respectively. The figures show that the curves are almost overlapping each other and the difference between them is not visible. Therefore, it can be said that the metal frame has no significant effect on the radiation patterns of the antenna.

\section{CONCLUSIONS}

An experimental study for the feasibility of installation of solar panels on two designated areas on a short-wave radio transmitter station is carried out via on-site measurements and computer simulations and the results are compared with the human exposure limits of ICNIRP. Effects of the metallic frame of solar panel on the radiation patterns of the antenna are also studied. In terms of ICNIRP 
human exposure limits, the two regions of interest are not recommended for solar panel installation. In the yellow region, the measured and simulated $E$-field values at some points are greater than human exposure limits. In redshaded region, the simulated and measured $E$-field results are below the human exposure limits. On the basis of simulation and the statistical nature of measurements, the redshaded region is also not recommended for solar panel installation. Moreover, the radiation patterns of the antennas are not affected significantly by the metal frames of the solar panels in the two designated areas.

The results deduced from this work are expected to be helpful for analogous EMC studies regarding the effects of metal-frame structures in the near-field of high-power $\mathrm{HF}$ antenna arrays, and give beneficial information about the feasibility of construction of these structures in terms of their effects on the human health and electromagnetic radiation characteristics.

\section{ACKNOWLEDGMENT}

The authors would like to thank Tubitak (The Scientific and Technological Research Council Of Turkey) under the scholarship scheme 2215 and TRT for their financial support to produce this work.

\section{REFERENCES}

1. ICNIRP, Guidelines on limits of exposure to time-varying electric, magnetic and electromagnetic fields $(1 \mathrm{~Hz}$ to 300 GHz), Health Phys 74 (1998), 494-522.

2. ICNIRP, Exposure to high frequency electromagnetic fields, biological effects and health consequences $(100 \mathrm{kHz}$ to $300 \mathrm{GHz}$ ), Oberschleißheim, Germany, 2009.

3. ICNIRP, Guidelines on limiting exposure to time-varying electric and magnetic fields (1 Hz to $100 \mathrm{kHz})$, Health Phys 99 (2010), 818-836.

4. N. Cherry, Health Effects in the vicinity of Radio/TV towers and mobile phone base stations, Dept. Human Sci., Lincoln Univ., Canterbury, NZ, Rep., 2002.

5. Y. Shimizu, K. Shigeta, K. Yukawa, T. Nakamura, M. Mikkaichi, Y. Nagasawa, and R. Sato, Analysis of skew antenna around a square tower for UHF TV broadcasting, IEEE Trans Antennas Propag 36 (1988), 927-935.

6. A. Ali, Analysis and design of broadcast tower antenna systems, M.S. thesis, Dept. Elect. \& Electron. Eng., Bilkent Univ., Ankara, Turkey, 2014.

7. W. Sheng and L. Erping, Simulation of a communication antenna system electromagnetic radiation and coupling, International Conference on Microwave and Millimeter Wave Technology Proceedings, Beijing, China, 2000, pp. 363-366.

8. A. Voors, NEC based antenna modeler and optimizer, [Online]. 2005. Available from: http://www.qsl.net/4nec2/. Last accessed on 1 July 2015.

9. C.A. Balanis, Antenna Theory, Analysis and Design, 3rd ed., Wiley, New Jersey, 2005, Chapter 2, sec. 13, pp. 80-85.

\section{BIOGRAPHIES}

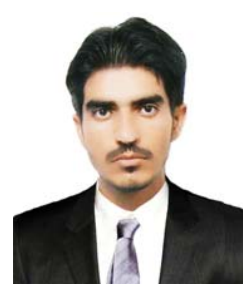

Abdul Ali was born in Quetta, Pakistan, in 1987. He received the B.S. degree in telecommunication engineering from National University of Computer and Emerging Sciences, Peshawar, Pakistan, in 2011 and the M.S. degree in electrical and electronics engineering from Bilkent University, Ankara, Turkey, in 2014, where he is currently pursuing the Ph.D. degree. His research interests include antenna design at high frequency, microwave circuits, and integrated circuits.

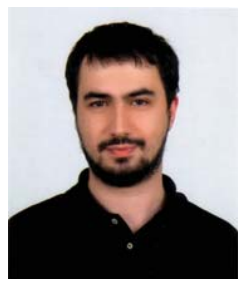

Burak Ozbey was born in Ankara, Turkey, in 1986. He received the B.S. and M.S. degrees in electrical and electronics engineering from Bilkent University, Ankara, Turkey, in 2008 and 2011, respectively, where he is currently pursuing the Ph.D. degree. His research interests include electromagnetic theory, microwave circuits, metamaterials, and design and testing of wireless RF sensors.

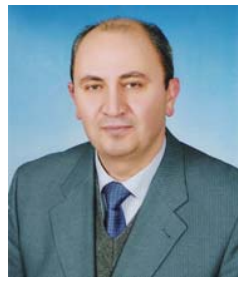

Satilmis Topcu was born on August 7, 1964 in Ankara, Turkey. He received the B.Sc. degree in electrical and electronics engineering from Middle East Technical University in 1986. He received the M.Sc. and Ph.D. degrees in electrical and electronics engineering from Bilkent University in 1989 and 1994. He became a faculty member in the Department of Electrical and Electronics Engineering, Eastern Mediterranean University in Cyprus from 1995 to 1998. He joined the Communications and Spectrum Management Research Center of Bilkent University in 1998 and he is presently assistant director. He has also been a faculty member in the Department of Computer Technology and Information Systems in Bilkent University since 2007. His research interests include development of advanced automated spectrum management systems, propagation prediction, network and frequency planning for wireless communication systems as well as the design, simulation, and testing of VLSI circuits. Dr. Topcu is a member of IEEE.

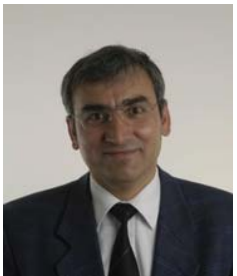

Ayhan Altintas received the B.S. and M.S. degrees from Middle East Technical University (METU), Ankara, Turkey, in 1979 and 1981, respectively, and the Ph.D. degree from The Ohio State University, Columbus, in 1986. From 1981 to 1987, he was with the ElectroScience Laboratory, The Ohio State University. He is currently a Professor of Electrical Engineering with Bilkent University, Ankara, Turkey. He has held research fellow and guest professor positions at Australian National University, Canberra, Australia, the Tokyo Institute of Technology, Japan, the Technical University of Munich, Germany, and Concordia University, Montreal, Canada. His research interests include high frequency and numerical techniques in electromagnetic scattering and diffraction, propagation modeling and simulation, and fiber and integrated optics. He is a member of Sigma Xi and Phi Kappa Phi. He is a Fulbright Scholar, and an Alexander von Humboldt Fellow. He is the President of the URSI Turkish National Committee. 